\title{
LATTICE FUNCTION MEASUREMENT OF FNAL MAIN INJECTOR AT 8-GEV ${ }^{\dagger}$
}

\author{
S. Assadi, C.S. Mishra, F. Tecker, M.J. Yang ${ }^{\ddagger}$, Fermilab
}

\begin{abstract}
The commissioning of Fermilab Main Injector had been very successful and the project is at its final phase. Even with the machine working as well as it did plenty of improvements are still to be made. Measuring and to understanding the machine lattice function should eventually lead to the improvement of the machine. In this write-up the preliminary measurement and analysis of the lattice function is reported.
\end{abstract}

\section{INTRODUCTION}

The Main Injector is a newly commissioned machine. Although effort was made to ensure that it performs as designed the possibility of magnetic field error is always there. It is important to know the actual lattice function in order to improve the machine performance. It is needed to properly match the $8-\mathrm{GeV}$ transfer line to the ring, to assess possible aperture restriction, and to design extraction transfer line optics. Comparing the measurement to the design will help locating the origin of errors.

The effort to measure the lattice function of Main Injector is still at its early stage. A measurement at 8$\mathrm{GeV}$ injection energy is an important first step. This write-up reports the preliminary analysis result from data taken during the commissioning run in the February of 1999 and is presented as a progress report. More measurement undoubtedly will be made in the very near future to validate the result.

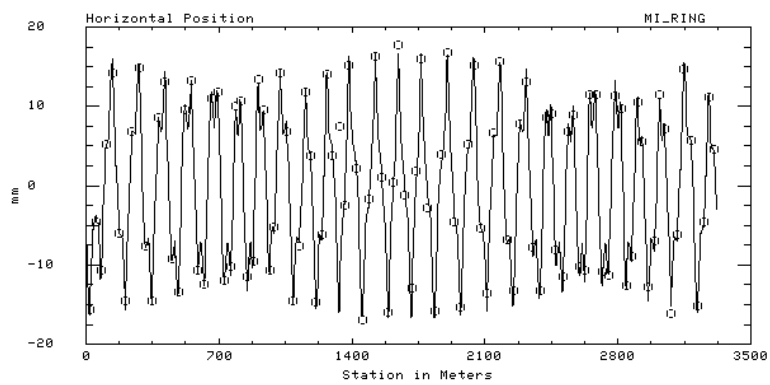

Figure 1. The horizontal difference orbit caused by 1-bump kick at the corrector H104, which was set 2.5 amp lower.

\section{DATA}

There are a total of 208 BPMs around the Main Injector ring, 104 horizontal plane BPMs at focusing locations and 104 vertical plane BPMs at de-focusing locations. The reading from all were collected during study at any one data sample. Along with that are the currents for bend bus, quad bus, and trim correctors. The RF frequency and

\footnotetext{
$\dagger$ Work supported by the US Department of Energy under contract DE-AC02-76CH00300.
}

* E-mail: YANG@FNAL.GOV intensity reading are also included. This was accomplished using the on-line analysis program [1] and analyzed.

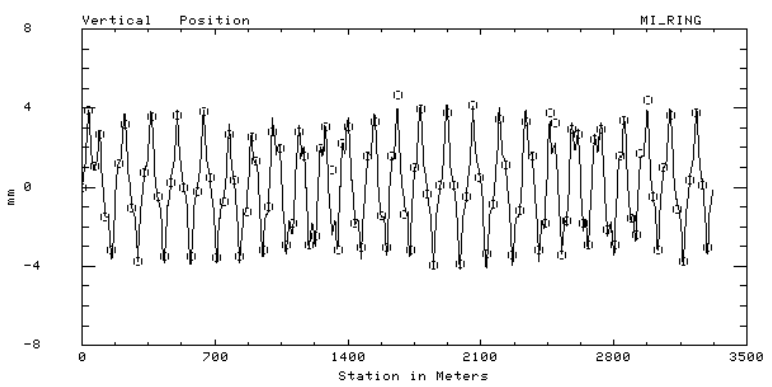

Figure 2. The vertical closed orbit due to 1-bump kick from corrector V105 that was set to 1.5 amps above nominal.

To do the lattice function measurement two dipole correctors from each plane were chosen. The design phase advance between the two correctors is close to 90 degree in either plane. Shown in Figure 1 is an example of horizontal plane orbit data taken with $\mathrm{H} 104$ corrector. In Figure 2 is an example in the vertical plane using corrector V105. With each corrector a series of BPM orbit data were collected, with corrector current changed in steps of 0.5 Amps. This corresponds to about $120 \mu$ rad in horizontal kick for $\mathrm{H} 104$ and $\mathrm{H} 106$ correctors at $8-\mathrm{GeV}$ energy. The vertical correctors are weaker and the kick was only about $50 \mu \mathrm{rad}$ for V105 and V107. Figure 3 shows a plot of BPM position reading at MI 122 location as a function of the kick angle from H104. Figure 4 shows the vertical position at VP407 as a function of kick angle from V105. The slope of linear least square fit to the data points will be used for subsequent analysis.

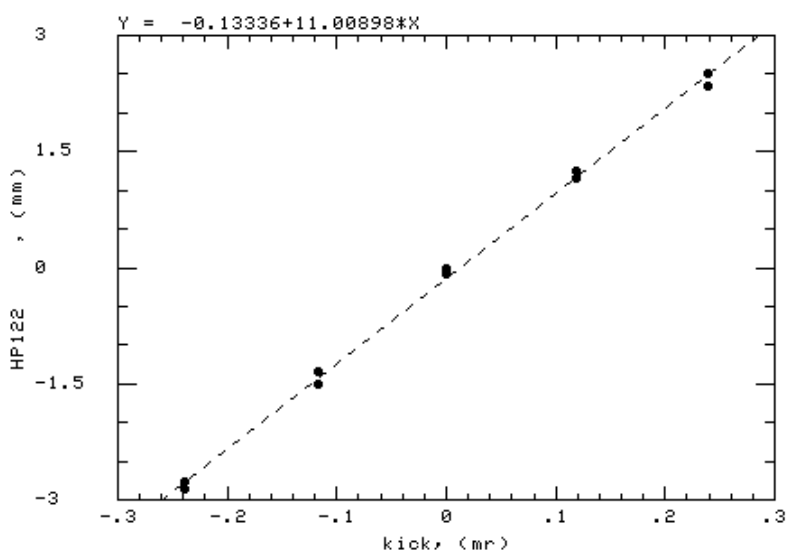

Figure 3. The orbit data taken at BPM HP122. Horizontal corrector $\mathrm{H} 104$ current was changed in step of $0.5 \mathrm{amps}$. The result of least square fit to the data points is shown at the top and plotted in dashed line. 
In order to measure the dispersion function the Main Injector RF frequency was changed at $250-\mathrm{Hz}$ steps, about $0.053 \%$ in $\bullet p / p$. Both horizontal and vertical data were taken for analysis. Figure 5 is an example of data taken for dispersion function measurement at HP208 location.

\section{ANALYSIS}

The lattice function analysis is based on the equation:

$$
x_{i}^{(k)}=\frac{-\sqrt{\beta_{i} \beta^{(k)}} \cdot \Delta \theta^{(k)} \cos \left[2 \pi\left(\psi_{i}-\psi^{(k)} \mid-Q / 2\right)\right]}{2 \cdot \sin (\pi Q)}
$$

The index $k$ denotes the corrector element used and $i$ the BPM index. The symbol $Q$ is the machine tune and $\psi$ the phase advance in units of $2 \pi$. This equation can be rewritten as:

$$
y_{i}^{(k)}=\frac{-\sqrt{\beta_{i} \beta^{(k)}} \cdot \cos \left[2 \pi\left(\psi_{i}-\alpha^{(k)}\right)\right]}{2 \cdot \sin (\pi Q)}
$$

Where $y_{i}^{(k)}=x_{i}^{(k)} / \Delta \theta^{(k)}$ is the slope in the example shown in Figure 3 or Figure 4.

And $\alpha^{(k)}=\psi^{(k)} \pm Q / 2$, the adjusted corrector phase. The negative sign is needed when $\psi_{i}<\psi^{(k)}$.

With two sets of corrector data the equations for the phase advance can be written:

$$
\begin{aligned}
& \cos \left(2 \pi \psi_{i}\right)=\frac{+2 \sin (\pi Q) \cdot\left[\sqrt{\beta^{(2)}} y_{i}^{(1)} \sin \left(2 \pi \alpha^{(2)}\right)-\sqrt{\beta^{(1)}} y_{i}^{(2)} \sin \left(2 \pi \alpha^{(1)}\right)\right]}{\sqrt{\beta_{i} \beta^{(1)} \beta^{(2)}} \sin \left(\alpha^{(1)}-\alpha^{(2)}\right)} \\
& \sin \left(2 \pi \psi_{i}\right)=\frac{-2 \sin (\pi Q) \cdot\left[\sqrt{\beta^{(2)}} y_{i}^{(1)} \cos \left(2 \pi \alpha^{(2)}\right)-\sqrt{\beta^{(1)}} y_{i}^{(2)} \cos \left(2 \pi \alpha^{(1)}\right)\right]}{\sqrt{\beta_{i} \beta^{(1)} \beta^{(2)}} \sin \left(\alpha^{(1)}-\alpha^{(2)}\right)}
\end{aligned}
$$

Using these two equations the phase advance $\psi$ at the $i$-th BPM location can be calculated. With phase advance from either data or from model the beta function can be calculated using Equation (2).

The analysis of the dispersion function requires the knowledge of the momentum error. This is calculated from the RF frequency recorded during the data taking using the equation:

$$
\frac{\Delta p}{p}=-\eta \frac{\Delta f}{f},
$$

Where the phase slip factor $\eta$ defined in $\frac{1}{\eta}=\frac{1}{\gamma_{t}^{2}}-\frac{1}{\gamma^{2}}$

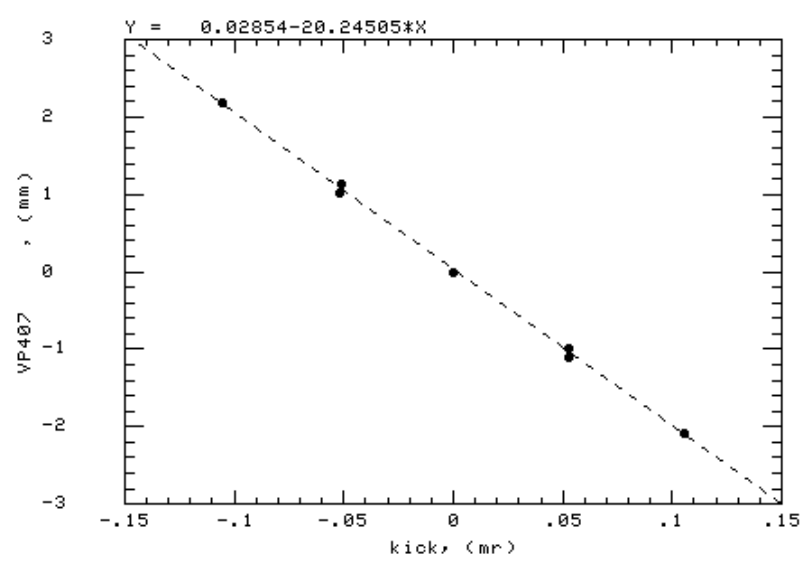

Figure 4. Vertical plane position data at VP407 as a function of kick angle from vertical corrector V105, at every $0.5 \mathrm{amps}$.

\section{RESULTS}

The analysis of the phase advances, beta function, and the dispersion function will be reported here. The statistical error analysis was performed and displayed with the plotted data points. The work is just starting to sort out systematic errors that could affect the analysis, i.e. beta, phase, machine tune, the corrector kick strength, the phase slip factor.



Figure 5. Horizontal beam position at location 208 as a function of the momentum error.

\subsection{Phase advances}

Figure 6 shows the deviation of the measured phase advances from that of model calculation, for both plane. The phase advance analysis is in principle immune to the BPM calibration error. The RMS deviation as shown in the plot amounts to about 4 degrees of error.


Figure 6. This is a plot of differences between the measured phase advances and phase advances calculated from model. The quantity displayed is in unit of $2 \bullet$. The lower plot is for the horizontal plane and upper plot for vertical plane. 


\subsection{Betafunction}

The beta function, on the other hand, is dependent on the calibration of the BPM signal, along with all other assumptions that go into the analysis. Figure 7 shows the measured beta function along with the model calculation. The fractional deviation from the model is plotted in Figure 8. In both plot the horizontal plane is shown at the bottom and vertical at the top.

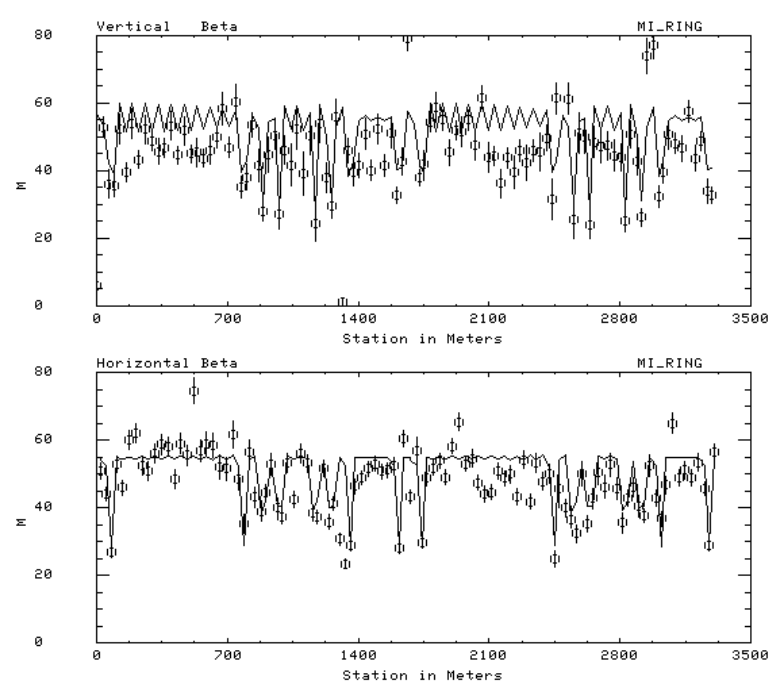

Figure 7. The measured Main Injector beta functions are shown here in green circles. The calculated beta functions, at the corresponding BPM locations, are shown in solid line.

\subsection{Dispersion function}

Dispersion-free regions are part of Main Injector design and are important to the function of the machine. This is confirmed readily from the data in part because it requires only null result, i.e. no position movement as function of energy error.


Figure 8. The horizontal plane beta function errors are shown in the lower plot and the vertical plane errors in the upper plot. The vertical axis is the fractional deviation of the measured beta function with respect to the calculated values.
The measured dispersion functions in both horizontal and vertical plane are shown in Figure 9. No vertical dispersion function is apparent, as expected. There are, however, plenty of locations where the dispersion function deviates from the design value by as much as 0.4 meters.

The negative dispersion function seen on the plot is because Main Injector was designed to runs counter clockwise with proton. For a right-handed coordinate system positive horizontal direction is toward inside of machine. Beam particles with larger momentum will move toward outside, i.e. the negative direction.
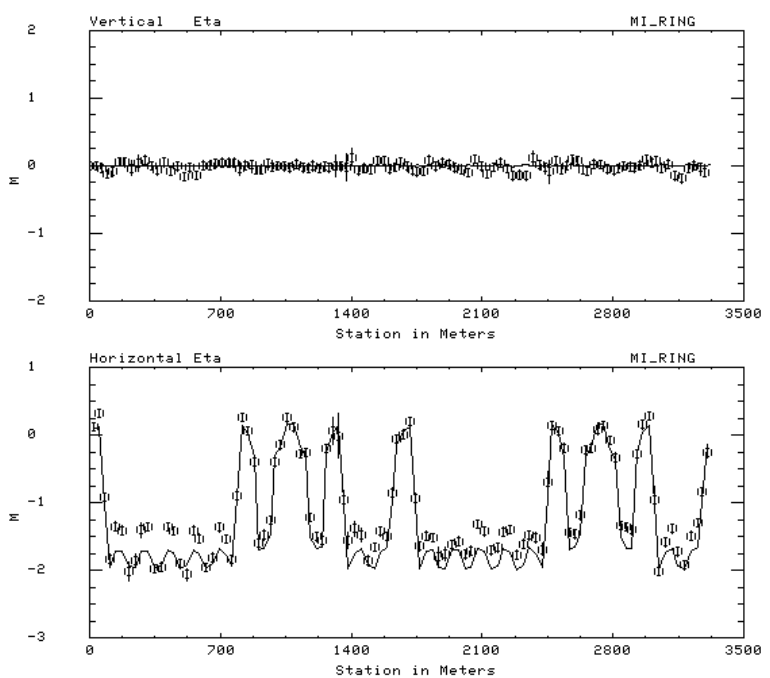

Figure 9. The measured dispersion functions are plotted in green circles and the calculated dispersion functions at the corresponding BPM locations are shown in solid line.

\section{CONCLUSION}

The analysis result shown here is considered very preliminary. More measurement in the future will no doubt shed more light on the consistency of measurement.

During commissioning the BPMs have worked well. The measurement of lattice function, however, might require a level of precision that is yet to be understood. Cross-plane signal coupling is a known issue for the Main Injector BPMs. Resolution and calibration measurement using beam data is still to be done.

The measured phase advances around the Main Injector ring were not far from the design values. The measured beta function in the vertical plane appears to deviate more significantly. The fact that data appears to follow the variation of the design suggests some credibility. The measured dispersion function is another indication of imperfection in the existing lattice and that can not be explained as from BPM error.

\section{REFERENCES}

[1] M.J. Yang, "Beam Analysis program for Main-Injector Commissioning." this proceeding. 